\title{
Construção de uma linha de cuidado para atenção à saúde de mulheres vivendo com HIVa
}

\author{
Construction of a line of care for the health care of women living with HIV \\ Construcción de una línea de cuidado para atención a la salud de mujeres viviendo con VIH
}

\begin{abstract}
Raquel Einloft Kleinubing ${ }^{1}$ (D) Tassiane Ferreira Langendorf ${ }^{2}$ (1) Stela Maris de Mello Padoin ${ }^{2}$ (I) Cristiane Cardoso de Paula ${ }^{2}$ (D)
\end{abstract}

1. Universidade Federal de Santa Maria, Programa de Pós-Graduação de Enfermagem. Santa Maria, RS, Brasil.

2. Universidade Federal de Santa Maria, Departamento de Enfermagem. Santa Maria, RS, Brasil.
Autor correspondente:

Cristiane Cardoso de Paula.

E-mail: cristiane.paula@ufsm.br

Recebido em 04/03/2021.

Aprovado em 06/07/2021.

DOI:https://doi.org/10.1590/2177-9465-EAN-2021-0033

\section{Resumo}

Objetivo: Construir uma linha de cuidado para a atenção à saúde de mulheres vivendo com HIV. Método: Pesquisa participante desenvolvida por meio da técnica de grupo focal com 11 profissionais e gestores de serviços de saúde em município do Sul do Brasil. Os dados foram analisados a partir da análise de conteúdo. Resultados: Os profissionais e gestores elencaram dificuldades e facilidades para promoção do acesso das mulheres que vivem com HIV aos serviços de saúde. Identificaram a descentralização do cuidado, os serviços articulados em Redes de Atenção à Saúde e a constituição da linha de cuidado para mulheres vivendo com HIV como estratégia para potencializar a qualidade da atenção à saúde para essa população. Conclusão e implicações para a prática: Considera-se que a linha de cuidado deve ser flexível, horizontal e levar em consideração escolhas e autonomia das mulheres no acesso aos serviços de saúde. A construção desse produto, mediado pela participação efetiva de profissionais e gestores em pesquisa participante, indica o potencial para a reorganização dos serviços em rede.

Palavras-chave: Assistência Integral à Saúde; Acesso aos Serviços de Saúde; HIV; Saúde da Mulher; Saúde Materno-Infantil.

\section{Abstract}

Objective: To build a line of care for the health care of women living with HIV. Method: Participatory research developed through focus groups with 11 professionals and health service managers in a city in southern Brazil. Content analysis was applied to the data collected. Results: Professionals and managers listed difficulties and facilities to promote access to health services for women living with HIV. They identified the decentralization of care, the articulation of services in Health Care Networks and the constitution of the line of care for women living with HIV as strategies to enhance the quality of health care for this population. Conclusion and implications for practice: It is considered that the line of care must be flexible, horizontal and take into consideration choices and autonomy of women in the access to health services. The construction of this product, mediated by the effective participation of professionals and managers in this research, indicates the potential for reorganization of network services.

Keywords: Comprehensive Health Care; Health Services Accessibility; HIV; Women's Health; Maternal and Child Health.

\section{RESUMEN}

Objetivo: Construir una línea de cuidado para la atención a la salud de las mujeres viviendo con VIH. Método: Investigación participativa desarrollada mediante la técnica de grupo focal con 11 profesionales y gestores de servicios de salud en un municipio en el Sur de Brasil. Se aplicó el análisis de contenido a los datos producidos. Resultados: Los profesionales y gestores enumeraron dificultades y facilidades para promoción del acceso de las mujeres que viven con el VIH a los servicios de salud. Identificaron la descentralización del cuidado, los servicios articulados en las Redes de Atención a la Salud y la constitución de la línea de atención a las mujeres viviendo con VIH como estrategias para mejorar la calidad de la atención a la salud de esta población. Conclusión e implicaciones para la práctica: Se considera que la línea de atención debe ser flexible, horizonta y tener en cuenta las opciones y la autonomía de las mujeres en el acceso a los servicios de salud. La construcción de este producto, mediada por la participación efectiva de profesionales y gestores en la investigación participativa, indica el potencia para la reorganización de los servicios de la red.

Palabras clave: Atención Integral de Salud; Accesibilidad a los Servicios de Salud; VIH; Salud de la Mujer; Salud Materno-Infantil. 


\section{INTRODUÇÃO}

As doenças relacionadas com a infecção pelo vírus da imunodeficiência humana (HIV) continuam sendo a principal causa de morte entre mulheres em idade reprodutiva (1549 anos) em todo o mundo, apesar da cobertura e da adesão ao tratamento contribuírem para o contínuo declínio das mortes relacionadas à aids ${ }^{1}$. E, essa situação poderá ocorrer durante o período gravídico-puerperal, o que implica na necessidade de mudanças na organização e oferta de atenção à saúde da mulher que vive com HIV, especialmente durante a gestação².

O diagnóstico da infecção pelo HIV em mulheres ocorre, na maioria das vezes, durante a gestação, e implica em ampliar o rastreio do vírus a partir de ações desenvolvidas pelos serviços de saúde ancoradas em políticas como a Rede Cegonha. Esta tem entre as estratégias assegurar o acesso aos serviços de saúde e a cobertura da testagem rápida para o HIV e outros agravos em gestantes e seus parceiros na Atenção Primária à Saúde (APS), entendendo que é de competência das equipes de saúde a realização de testes rápidos durante a assistência pré-natal ${ }^{3,4}$.

Em outros países, assim como no Brasil, a atenção pré-natal consiste em um momento chave para intensificação do rastreamento, uma vez que durante a consulta deverá ter espaço para a orientação quanto à importância da testagem e os benefícios à saúde maternoinfantil, no que tange o diagnóstico precoce, tanto para o controle da infecção materna quanto para a prevenção da transmissão vertical do HIV5,6. Para tanto, exige-se do Sistema Único de Saúde (SUS) serviços suficientes e articulados em Rede de Atenção à Saúde (RAS). A operacionalização da RAS depende da articulação entre os seus elementos constitutivos: população/região de saúde definidas, estrutura operacional e sistema lógico de funcionamento determinado pelo modelo de atenção à saúde ${ }^{7}$.

Atualmente, a RAS está voltada para o atendimento de condições agudas, mas deve ser um sistema capaz de enfrentar as novas demandas que surgiram com a intensificação das condições crônicas, dentre elas, a infecção pelo HIV ${ }^{8}$. Para a efetivação da RAS, deverá garantir o acesso aos serviços como um dos seus pilares, além de garantir que se desenvolva a coordenação do cuidado. Esse é um atributo da qualidade da atenção dos serviços de saúde que deverá ser capaz de não somente direcionar o usuário dentro da rede, mas sim coordenar oportunamente o trajeto percorrido entre os diferentes pontos de cuidado $^{9}$. Apesar dos serviços de APS representarem a porta de entrada preferencial no sistema de saúde para as pessoas vivendo com HIV, inclusive às gestantes, o acesso e coordenação do cuidado são ações ainda insuficientes ${ }^{10}$.

Em estudo desenvolvido na região sul do Brasil, $74,4 \%$ das participantes que eram gestantes vivendo com HIV referiu como fonte regular de atenção à saúde os serviços de APS. Essas mulheres consideram que a APS foi o serviço que melhor as conhecia e aquele que tinha maior responsabilidade por sua saúde durante a gestação. Tais questões definiram o grau de afiliação ao serviço de APS, e as usuárias consideraram que a qualidade da atenção recebida foi insatisfatória tanto na atenção primária $(6,50)$ quanto na especializada $(6,35)^{11}$.
Para que seja possível superar as barreiras de acesso e de comunicação entre os serviços de saúde que, muitas vezes, consistem em entraves para o funcionamento da RAS às pessoas com HIV, a implantação de uma linha de cuidado (LC) poderia atuar como uma eficiente estratégia. LC é uma estratégia, em resposta às necessidades epidemiológicas, para articular recursos e práticas de produção de saúde entre os pontos de atenção, visando a condução oportuna, ágil e singular dos usuários para o diagnóstico e a terapia ${ }^{7}$.

Apesar de essa estratégia ser indicada em diretrizes nacional ${ }^{7}$ e estadual ${ }^{12}$, há lacuna na construção de uma LC que considere as especificidades locais e epidemiológicas deste agravo nesta população, bem como de oferta de serviços de saúde. Uma LC adaptada ao contexto local pode auxiliar os profissionais de saúde no direcionamento das ações e na tomada de decisão, considerando o percurso dessa população na RAS e as possibilidades de transferências entre esses serviços. Assim, a questão de pesquisa "como desenvolver, de modo participativo com os tomadores de decisão, uma estratégia que promova o acesso aos serviços de saúde no município?" culminou no objetivo de construir uma LC para a atenção à saúde de mulheres vivendo com HIV.

\section{MÉTODO}

Estudo de abordagem qualitativa, do tipo participante. Foi guiado pelo Modelo de Tradução do Conhecimento em Ação ${ }^{13,14}$, que pretende que os resultados de pesquisa ultrapassem a disseminação entre os pares, promovendo o intercâmbio de conhecimentos de modo participativo por meio da criação e aplicação de ferramentas para qualificar a atenção e assim preencher a lacuna entre o que se sabe e o que se faz.

O modelo possui um ciclo de criação do conhecimento e um de ação. Esta pesquisa se localizou no ciclo de criação, que contempla as fases: averiguação do conhecimento existente, desenvolvida por meio de observação participante nos serviços de saúde e de entrevistas com as mulheres vivendo com HIV; síntese do conhecimento, desenvolvida com a triangulação dos dados da pesquisa ${ }^{15}$ e de um estudo de revisão ${ }^{16}$; e ferramentas/ produtos do conhecimento, desenvolvida no grupo focal (GF). (Figura 1). A última fase é o foco desse artigo.

A produção de dados foi desenvolvida em município da região central do Estado do Rio Grande do Sul (RS), Brasil. Nesse município, a atenção à saúde das mulheres vivendo com HIV é ofertada por meio das redes de atenção primária e especializada. A APS tem 31 serviços, dos quais 18 são Unidades Básicas de Saúde tradicionais (UBS) e 13 são Estratégia Saúde da Família (ESF). O serviço especializado é um Hospital Universitário de âmbito federal e um ambulatório municipal.

Os critérios de inclusão foram: ser gestor ou profissional da saúde com atuação nos serviços de APS ou especializado, prestando ações de atenção à saúde da mulher. Àqueles não pertencentes ao quadro efetivo do município ou em atestado de saúde ou afastamento do trabalho no período de coleta de dados foram excluídos. Participaram do GF três gestores da Secretaria de Saúde do Município e oito profissionais de serviços de APS 


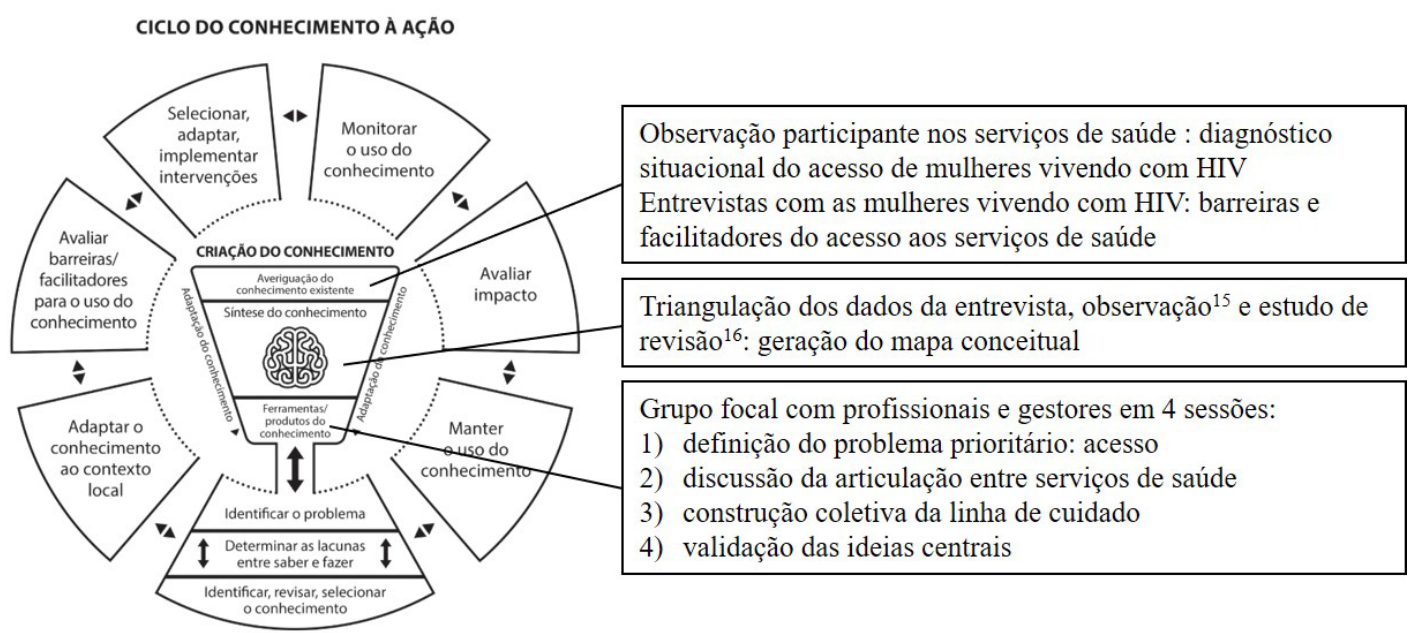

Figura 1. Percurso da pesquisa participante com profissionais e gestores na elaboração da linha de cuidado para mulheres vivendo com HIV. Brasil, 2018.

Fonte: adaptado de Vieira et al..$^{14}$

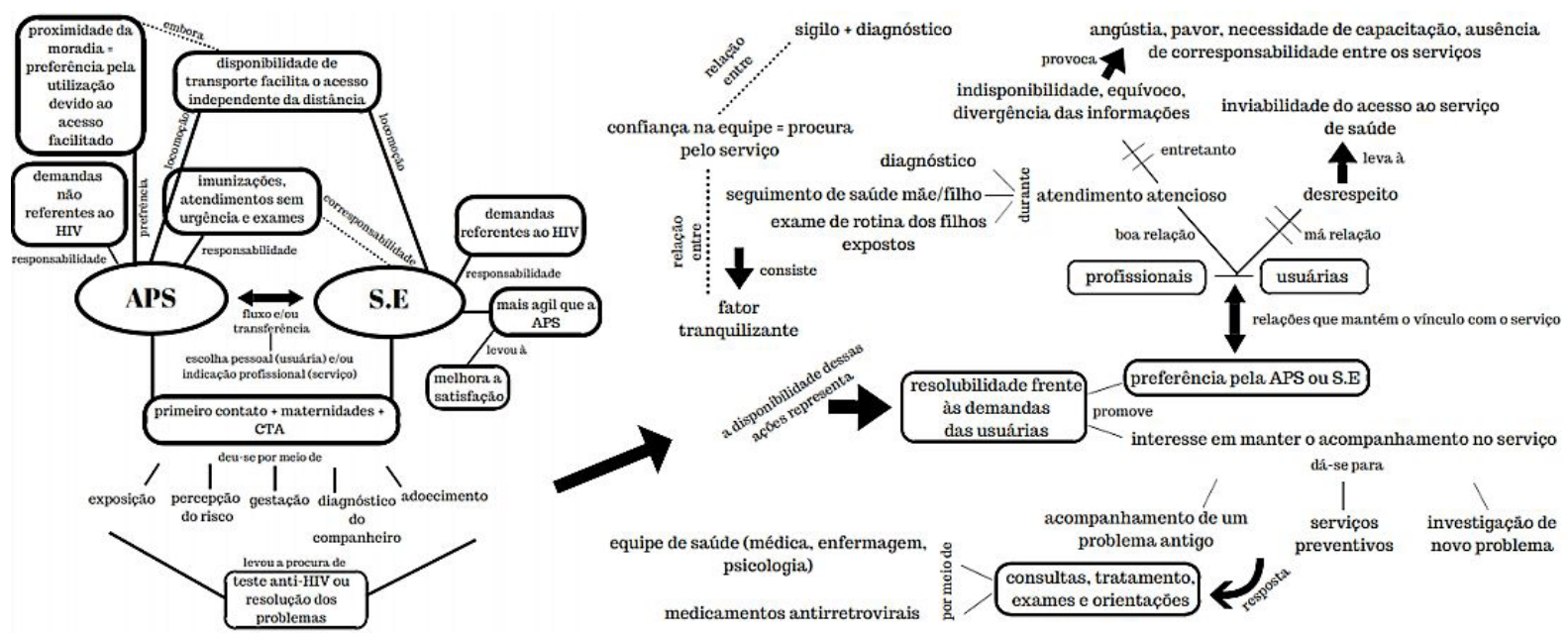

Figura 2. Mapa conceitual apresentado aos participantes para guiar as discussões na 1a sessão de grupo focal. Brasil, 2018. Legenda: $A P S=$ serviços de atenção primária a saúde; $S E$ = serviços especializados

Fonte: elaborada pelas autoras, 2018.

e do serviço especializado do município, com representação de enfermeiros, psicólogos e Agentes Comunitários de Saúde (ACS).

$\mathrm{Na}$ etapa de recrutamento dos participantes, foi enviado um convite formal para os serviços e foi agendada reunião para definir a melhor estratégia para os encontros do GF. Foi criada uma lista (nome/contato) daqueles profissionais interessados e que atendessem aos critérios de inclusão.

A produção de dados foi por meio da técnica de $\mathrm{GF}^{17} \mathrm{com} \mathrm{a}$ realização de quatro sessões, com intervalo de aproximadamente um mês entre as mesmas, entre maio a outubro de 2018. O quantitativo de participantes variou por sessão, e não houve recusa e nem desistências durante a produção de dados. $\mathrm{Na}$ abertura de cada sessão, foi realizado o acolhimento dos participantes e o acordo com o grupo acerca do compromisso ético e da operacionalização ou dinâmica do GF, e o objetivo da pesquisa foi apresentado. A equipe de coordenação foi composta pela moderadora e por duas observadoras com experiência no tema de HIV e APS, bem como na técnica grupal. Foi feito registro em diário de campo e a duração de cada sessão foi de aproximadamente duas horas.

Na primeira sessão, foi definido o problema prioritário a ser discutido, e então foram apresentados resultados de pesquisa anterior (Figura 2) para guiar as discussões acerca do acesso das mulheres aos serviços de saúde no município. O tema foi definido pelo grupo.

O debate foi mediado por questões norteadoras (Quadro 1) e a cada sessão foi realizada uma síntese. A segunda sessão foi de discussão da articulação entre serviços de saúde. Na terceira, 

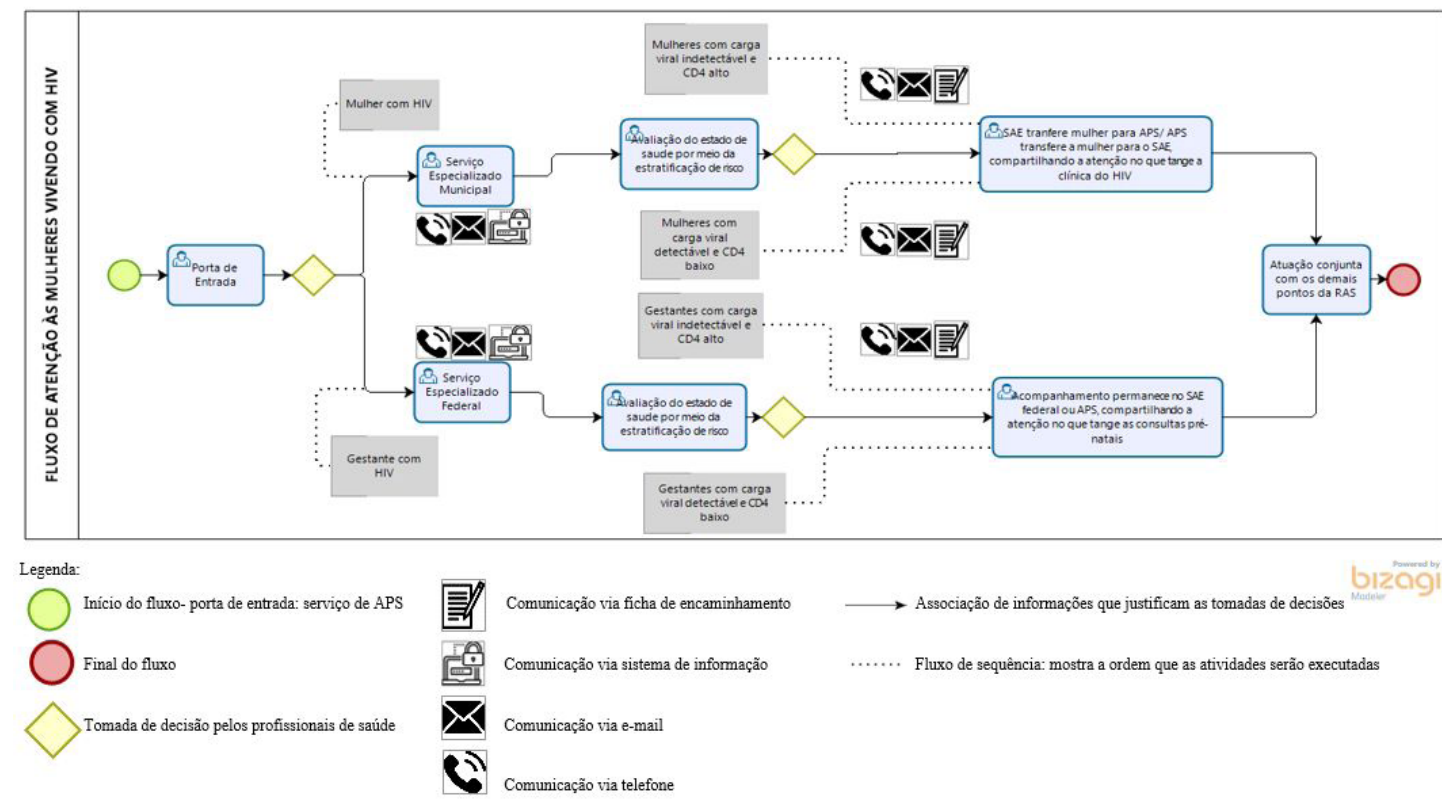

Figura 3. Linha de cuidado para mulheres vivendo com HIV. Brasil, 2018.

Fonte: elaborada pelas autoras, 2018.

Quadro 1. Questões disparadoras do grupo focal com profissionais e gestores. Brasil, 2018.

\begin{tabular}{|cc|}
\hline SESSÃO & QUESTÕES DISPARADORAS DE DEBATE \\
\hline $\mathbf{2}$ & $\begin{array}{c}\text { O que vocês compreendem por RAS? E, acerca de LC e } \\
\text { descentralização do cuidado? O que uma LC deve contemplar? }\end{array}$ \\
$\mathbf{3}$ & Como desenvolver a LC? \\
$\mathbf{4}$ & Validação das ideias centrais: LC para mulheres vivendo \\
com HIV.
\end{tabular}

Legenda: $R A S=$ rede de atenção à saúde; $L C=$ linha de cuidado

a LC foi desenvolvida coletivamente. E, na quarta, as ideias centrais foram retomadas e validadas na discussão participativa.

Por meio da interação entre pesquisadora e profissionais dos diferentes serviços que prestam atendimento às mulheres vivendo com HIV, o GF permitiu a construção coletiva dos resultados da pesquisa, que são representados pela elaboração da LC para mulheres vivendo com HIV (Figura 3), estruturando a RAS dessa população no município.

A análise de conteúdo do tipo temática ${ }^{18}$ foi desenvolvida em três fases: 1) a pré-análise consistiu na seleção inicial das notas transcritas das sessões do GF para constituição do corpus para análise e na pré-extração de unidades de significação;2) a exploração do material: as unidades de significação, previamente selecionadas, foram agrupadas e reagrupadas com o intuito de discutir a perspectiva de RAS para mulheres vivendo com $\mathrm{HIV}$; 3 ) para o tratamento dos dados e interpretação, os resultados foram retomados, aprofundados e discutidos com a literatura científica.

Respeitaram-se os preceitos éticos contidos na Resolução CNS 466/12. Os participantes tomaram conhecimento e assinaram o TCLE. A presente pesquisa foi aprovada pelo Comitê de Ética da Universidade Federal de Santa Maria, sob o Certificado de Apresentação para Apreciação Ética (CAAE) 57042216.0.0000.5346, parecer 1.635.237. Para manter o anonimato, foi utilizado o código NGF (Nota de Grupo Focal), seguido do número correspondente à sessão (1-4) e a letra $P$ (participante).

Ao final da pesquisa, foi enviado um ofício do Grupo de Pesquisa para a gestão municipal de saúde com o produto linha de cuidado e sua contextualização de produção.

\section{RESULTADOS}

A partir do mapa conceitual apresentado aos participantes para guiar as discussões na 1aㅡ sessão de GF (Figura 2), o grupo pactuou que o tema de discussão seria o atributo "acesso" da APS, no qual se tem o elemento estrutural "acessibilidade" e o elemento processual "utilização". Também foi discutido acerca do que poderia ser feito/quais ações poderiam ser desenvolvidas para promover a acessibilidade e utilização, e foram elencadas as dificuldades e facilidades apontadas pelos profissionais para a promoção do acesso.

[...] Elas [as mulheres vivendo com HIV] se sentem à vontade, acolhidas [no serviço de APS]. (NGF1 P1)

[...] A questão do acesso está bem relacionada com o acolhimento. (NGF1 P2)

[...] É importante o serviço especializado dar essa orientação [de que a mulher deve retornar para acompanhamento de rotina na APS] pra ela lá [no serviço especializado durante a gestação]. (NGF1 P7) 
E a gente tá falando só de [município em que a pesquisa foi desenvolvida], todos os municípios a gente vê essa dificuldade que ainda existe de perder o vínculo [comos serviços de APS]. (NGF1 P2)

[...] Eu acho que falta uma capacitação para os profissionais [de saúde] que não atenderam [as mulheres com HIV] nessas unidades [serviços de APS]. Que não precisam encaminhar tudo para a assistência especializada. (NGF2 P9)

As discussões da $2^{\underline{a}}$ sessão partiram da exposição da síntese do encontro anterior, por meio de uma apresentação de slides, e contemplaram questões sobre RAS, LC e descentralização do cuidado.

[...] Eu fiquei pensando na referência e contrarreferência. Como é bom quando a gente recebe a contrarreferência, tudo direitinho, das gestantes, quando ganham o bebê. (NGF2 P7)

[...] Com os protocolos tu vai saber que em qualquer lugar [serviço de saúde] vai ser atendido da mesma forma. [...] no curso de pré-natal que a gente fez eu vi que [...] a forma que eu atendo [no serviço de APS] é a mesma forma que elas atendem [no serviço especializado]. Elas também utilizam os protocolos do Ministério [da Saúde], não tem mistério, todo mundo vai atender da mesma forma como deve ser feito (NGF2 P7)

[...] Acho que [os profissionais de diferentes serviços de saúde] precisam falar a mesma língua, uma das coisas que eu acho importantes [...] [os serviços de saúde] ter contato, ter diálogo. (NGF2 P5)

A partir das discussões da $2^{\mathrm{a}}$ sessão, as participantes sugeriram que a moderadora buscasse articulação com a gestão municipal e os serviços municipais e federais para proposição de construção da LC para mulheres vivendo com HIV. Para tanto, foi elaborado um esboço dessa proposta. Com isso, o objetivo da $3^{2}$ sessão foi discutir com os profissionais dos serviços especializado e de atenção primária e gestores sobre como desenvolver a LC.

[...] articular os serviços, sentar junto com todos e conseguir definir estratégias, trabalhando todos juntos. Todos os niveis de atenção, pra poder construir, organizar, essa rede. Não é a gestão sozinha, não é o serviço especializado, não é a atenção básica, são todos. Eeu acho que é possível.[...] Eu acho que isso que se perdeu com o tempo, o sentar junto, o conversar, o dialogar, conseguir discutir o quê, os fluxos, como organizar tudo, pra melhorar o atendimento. (NGF1 P2)

[...] No serviço especializado federal não é o mesmo sistema [de comunicação] nosso [da APS], então a gente [profissionais de saúde da APS] não tem como saber [os desfechos das usuárias com HIV no SAEfederal]. (NGF3P4)

[...] As [gestantes] HIV que eram, pela Rede Cegonha, pela nota técnica de pré-natal, o HIV sozinho, se não tivesse nenhum outro problema, deveria ser atendido pela Atenção Básica, com acompanhamento especializado na Casa Treze. Só que não tem essa estrutura e tá indo tudo para o [SAE federal]. (NGF4 P3)

[...] A gestante vai para o [serviço especializado federal], mas volta pra unidade [APS]. Por mês, ela vai ter o dobro de consulta, [ela vai consultar] nos dois lugares. (NGF4 P4)

Na 4⿳亠丷厂 sessão, foi apresentada ao grupo para validação a versão final da LC para mulheres vivendo com HIV (Figura 3).

[...] [É necessário] os serviços [de saúde] se conversarem. (NGF2 P7)

[...] O serviço especializado federal tem que dar retorno pra unidade [serviço de APS] daquelas que não foram [...] tanto consulta de pré-natal, outras que tenham marcado, aqueles usuários que não estão pegando medicação. (NGF2 P3)

[...] Eu acho que não é a palavra "transferir" [na linha de cuidado], eu acho que é "compartilhada". Ela [mulher com HIV] volta pra atenção básica, mas ele [cuidado de saúde] é compartilhado. Ela vai para os dois. [...] Atendimento compartilhado com a Atenção Básica [e SAE]. (NGF4 P4)

[...] Nós da Atenção Básica, se é detectável ou indetectável a carga viral, ela [mulher] é positiva, ela vai ser compartilhada com o [SAE municipal]. E a gestante vai ser compartilhada com o [SAE federal] [...] seja gestante ou não gestante, independente da carga viral estar detectável, ou indetectável, a referência dela sempre vai ser a Atenção Básica. (NGF4 P4)

\section{DISCUSSÃO}

A descentralização da atenção às pessoas vivendo com HIV teve como uma de suas primeiras estratégias a realização do diagnóstico da infecção pelo HIV na APS, caracterizando esta como porta preferencial para testagem rápida, com intuito de ampliar as possibilidades de acesso. Entretanto, ao ampliar a gama de serviços habilitados a realizar a testagem anti-HIV, questões como o sigilo e revelação do diagnóstico podem gerar conflitos entre profissionais e usuários, o que pode implicar na fragilidade da relação de vínculo com o usuário ${ }^{19-21}$.

Para minimizar tal fragilidade, compreendemos que é necessário repensar a forma de organização e de gestão do trabalho nos serviços de saúde, que poderia envolver a discussão e implantação de uma estratégia como a LC, com vistas à qualificação da atenção às pessoas vivendo com HIV, bem como incluí-la na formação e educação permanente dos profissionais de saúde. Além disso, tal fragilidade se dá em razão de, muitas vezes, a assistência prestada a essa população estar pautada no conhecimento que o profissional adquire da sua experiência laboral, por meio de aprendizados informais e pelas trocas de informações com outros profissionais da equipe ${ }^{22}$. 
Considerando o cuidado à saúde na APS, sabe-se que este ponto de atenção possui potencial para o cuidado dos usuários vivendo com HIV, tanto quanto para outras condições crônicas de saúde, e contempla desde o diagnóstico até o tratamento. Para tanto, há necessidade de investimento para além das tecnologias leves, incorporando-se tecnologias diagnósticas e terapêuticas, bem como a articulação da APS com o serviço especializado ${ }^{10,23}$, configurando assim a LC como estratégia para condução dos usuários nos pontos de atenção à saúde.

A depender da atenção recebida, a escolha e predileção à continuidade do acesso ao serviço de saúde representam importantes indicadores de qualidade, pois refletem o grau de desempenho do serviço e de satisfação por parte de quem o acessa ${ }^{24}$. Além disso, devido aos profissionais de saúde representarem a rede secundária de apoio à essas mulheres, o acolhimento e o vínculo podem contribuir para essa escolha ${ }^{19,25}$. Ainda, a condução do cuidado às pessoas vivendo com HIV também pressupõe considerar as diferentes necessidades de saúde dessa população. Nesse sentido, devemos considerar o trabalho tendo como norte a LC com o envolvimento compartilhado dos diferentes pontos de acesso, em especial, da APS com o suporte dos serviços especializados ${ }^{26}$.

Isso implica na cooperação entre os serviços de saúde, característica relacionada à assistência integral à saúde, e remete à interdependência dos pontos, que são os serviços. Ou seja, entendese que nenhum serviço possui, de maneira isolada, a totalidade de recursos e competências para solucionar a diversidade de problemas de saúde da população que acessa uma rede de serviços ${ }^{27}$.

Dessa forma, a integração entre os serviços possibilitará o engajamento cooperativo e compartilhado das ações de saúde, e essa maneira de reorganizar os sistemas de atenção à saúde apresenta-se como uma estratégia para a qualificação do cuidado. Nessa perspectiva de atenção às pessoas vivendo com HIV, o SAE representa um ponto fundamental, porém não o único. Assim, o desenho da LC passa a contar com novos serviços de saúde e estratégias, como o suporte dos serviços especializados à APS e o atendimento compartilhado entre SAE e APS, garantindo maior acesso dos usuários ao sistema de saúde ${ }^{12-28}$.

Para tanto, a comunicação intersetorial será essencial para que se possa romper com a desarticulação, e para que a APS, em especial a ESF, possa se corresponsabilizar pela atenção a essa população. Isso significa, dentre outras atribuições, conhecer a população vivendo com HIV adstrita à unidade, responsabilizando-se pelo cuidado e pelo acompanhamento do tratamento. Dessa forma, o serviço especializado precisa estimular e orientar a utilização dos serviços de APS, tendo em vista que o grau de integração entre os serviços é decisório para a garantia do acesso ${ }^{29}$.

Acerca de recomendações nacionais para atenção à saúde materna e infantil, a Rede Cegonha se compromete com a detecção do HIV e com a Terapia Antirretroviral (TARV) durante a gestação, parto, puerpério e à criança exposta ao HIV. Entretanto, não há diretrizes que organizem de maneira específica uma LC às mulheres vivendo com HIV, tampouco no período gestacional. O estabelecimento de fluxos entre os pontos de atenção pode auxiliar gestantes vivendo com HIV a compreenderem a necessidade de acompanhamento compartilhado ${ }^{30}$ entre os pontos de atenção que integram a LC.

No âmbito estadual, a Secretaria de Estado da Saúde do RS, por meio da Coordenação Estadual de Infecções Sexualmente Transmissíveis (IST) e aids, elaboraram a "Linha de cuidado para pessoas vivendo com HIV/AIDS (PVHA) e outras IST". Com esse documento, apontaram diretrizes e recomendações gerais no intuito de subsidiar a definição de atribuições entre os pontos de atenção, buscando o compartilhamento do cuidado entre a APS, Centros de Testagem e Aconselhamento (CTA), Ambulatórios e Serviços de Atendimento Especializado em HIV/Aids (SAE) como tentativa de ofertar uma resposta mais adequada à epidemia ${ }^{12}$.

Entretanto, as gestantes vivendo com HIV não foram incluídas na estratificação, uma vez que o cuidado a essa população não está condicionado a critérios clínicos, ou seja, são situações específicas que necessitam de acompanhamento tanto na APS como pelo serviço especializado, independentemente da severidade do agravo. Isso revela a importância de se pensar em ações articuladas e condizentes, como a implementação da LC, com a realidade dos serviços com base nas possibilidades locais e considerando a participação fundamental dos profissionais de saúde que atuam nesses serviços, bem como dos gestores que coordenam as ações direcionadas a essa população ${ }^{12}$.

Com relação à APS, apesar do potencial para atender as gestantes vivendo com HIV, a falta de recursos humanos inviabiliza o acesso para o acompanhamento pré-natal ${ }^{25}$. Consideramos que, mesmo que haja o incentivo para que essa população acesse conjuntamente os serviços de APS e especializado, a incapacidade que a APS possui em suprir as demandas de saúde faz com que essas mulheres se vinculem a outros serviços, principalmente aos especializados ${ }^{31}$, fragilizando este como importante ponto da LC na atenção à saúde das mulheres vivendo com HIV. Além disso, entende-se relevante que o fluxo de transferência entre os serviços seja flexível, considerando-se a experiência e percepção do usuário quanto ao serviço recomendado. Assim, caberá ao usuário decidir qual local será o mais adequado para o acesso aos cuidados de saúde ${ }^{19}$.

Frente a isso, durante o período gravídico-puerperal, a APS deve responsabilizar-se pelo fornecimento de ações como aconselhamento, diagnóstico precoce de infecções, tratamento apropriado, ações educativas, orientações quanto ao tratamento e recomendações para a prevenção da transmissão vertical, encaminhamento dos casos não sensíveis a esse ponto de atenção e coordenação da rede ${ }^{32}$. Considerando-se a relevância da cooperação entre os serviços de saúde e o impacto positivo dessa estratégia de articulação, os participantes do estudo em tela construíram a LC para mulheres vivendo com HIV com o objetivo de promover o acesso e continuidade do cuidado dessa população aos serviços de saúde integrantes da RAS no município.

Tal iniciativa revelou existência de barreiras ao acesso, falta de coordenação do cuidado e do estabelecimento de fluxos formais e resolutivos. Tais barreiras consistem, por exemplo, na 
ausência de prontuários eletrônicos que integrem e compartilhem as informações clínicas entre os serviços ${ }^{33}$.

Com vista a superar esses desafios, entendemos a necessidade de que os diferentes pontos de atenção à saúde estejam articulados, favorecendo o trabalho em equipe entre os serviços. Entretanto, evidencia-se, como aponta um estudo que abordou a rede social de famílias de crianças com condições crônicas, a fragilidade no sistema de transferência entre as diferentes densidades tecnológicas, sobretudo à APS, a qual revela a desarticulação e a ausência de uma rede instituída nos municípios que possa superar essa falha operacional ${ }^{34}$. Ainda, evidencia-se que persiste a relação vertical entre profissionais de saúde e usuários, percebida pela falta da escuta e de orientações sobre o fluxo de acesso, bem como um tempo longo de espera pelo usuário para o atendimento especializado, o que implica na procura por diferentes meios de acesso aos serviços de referência ${ }^{35}$.

Há necessidade, também, de qualificação dos profissionais da APS para atuarem como coordenadores do cuidado, assim como o aperfeiçoamento do sistema de saúde no que tange a operacionalização de RAS integradas e articuladas. Para que isso seja possível, a APS necessita se articular com os diferentes pontos de atenção, garantir a porta preferencial de entrada e, com isso, ser resolutiva frente às necessidades de saúde da população ${ }^{31}$. Nesse contexto, a LC representa uma ferramenta organizativa que pode diminuir encaminhamentos desnecessários à atenção especializada, corrigir falhas de tecnologias de informação e comunicação, e substituir o uso de medidas informais de comunicação entre os profissionais.

A limitação da pesquisa consiste na necessidade de envolver mais profissionais da gestão e dos serviços especializados, uma vez que esses foram menos representados nos encontros grupais.

\section{CONSIDERAÇÕES FINAIS E IMPLICAÇÕES PARA A PRÁTICA}

Os participantes deste estudo discutiram as potencialidades para a articulação entre os serviços de saúde e definiram um percurso no sistema de saúde para mulheres que vivem com HIV, como uma linha de cuidado. Isso apontou a necessidade de instrumentos formais que assegurem as ações entre os diferentes pontos de atenção. Vislumbra-se a oportunidade da tomada de decisão na prática clínica pelos profissionais do modo compartilhado e conjunto. Destacamos a premissa de que esta linha deve ser flexível e levar em consideração a perspectiva das usuárias.

A construção desse produto, mediado pela contribuição efetiva de profissionais e gestores em pesquisa participante, indica o potencial para a reorganização dos serviços em rede. Torna-se imprescindível a corresponsabilização e compromisso da gestão municipal, uma vez que a implantação envolve mudanças estruturais e fortalecimento dos sistemas de saúde.

\section{AGRADECIMENTOS}

As autoras agradecem à Dra. Jaqueline Arboit e Doutoranda Jacqueline Silveira de Quadros, pelo apoio no desenvolvimento do grupo focal, como observadoras do tipo participante, na etapa de campo de produção de dados da presente pesquisa.

\section{FINANCIAMENTO}

O presente trabalho foi realizado com apoio da Coordenação de Aperfeiçoamento de Pessoal de Nível Superior - Brasil (CAPES) - Código de Financiamento 001, bolsa de doutorado concedido a Raquel Einloft Kleinubing.

\section{CONTRIBUIÇÕES DOS AUTORES}

Desenho do estudo. Raquel Einloft Kleinubing. Stela Maris de Mello Padoin. Cristiane Cardoso de Paula.

Coleta ou produção dos dados. Raquel Einloft Kleinubing.

Análise de dados. Raquel Einloft Kleinubing. Stela Maris de Mello Padoin. Cristiane Cardoso de Paula. Tassiane Ferreira Langendorf.

Interpretação dos resultados. Raquel Einloft Kleinubing. Stela Maris de Mello Padoin. Cristiane Cardoso de Paula. Tassiane Ferreira Langendorf.

Redação e revisão crítica do manuscrito. Raquel Einloft Kleinubing. Tassiane Ferreira Langendorf. Stela Maris de Mello Padoin. Cristiane Cardoso de Paula.

Aprovação da versão final do artigo. Raquel Einloft Kleinubing Tassiane Ferreira Langendorf. Stela Maris de Mello Padoin. Cristiane Cardoso de Paula.

Responsabilidade por todos os aspectos do conteúdo e a integridade do artigo publicado. Raquel Einloft Kleinubing. Tassiane Ferreira Langendorf. Stela Maris de Mello Padoin. Cristiane Cardoso de Paula.

\section{EDITOR ASSOCIADO}

Candida Primo Caniçali (1)

\section{EDITOR CIENTÍFICO}

Ivone Evangelista Cabral (D)

\section{REFERÊNCIAS}

1. Joint United Nations Programme on HIV/AIDS. UNAIDS data 2017 [Internet]. Geneva: UNAIDS; 2017 [citado 4 mar 2021]. Disponível em: https://www.unaids.org/sites/default/files/media_asset/20170720_ Data_book_2017_en.pdf

2. WHO: World Health Organization. UNICEF: United Nations Children's Fund. UNFPA: United Nations Population Fund. World Bank Group. United Nations Population Division. Trends in Maternal Mortality: 2000 to 2017: estimates by WHO, UNICEF, UNFPA, World Bank Group and the United Nations Population Division [Internet]. Geneva:WHO;2019 [citado 4 mar 2021]. Disponível em: https://www.unfpa.org/sites/default/ files/pub-pdf/Maternal_mortality_report.pdf

3. Ministério da Saúde (BR). Secretaria de Vigilância em Saúde. Departamento de Doenças de Condições Crônicas e Infecções Sexualmente Transmissíveis. Protocolo Clínico e Diretrizes Terapêuticas para Prevenção da Transmissão Vertical do HIV, Sífilis e Hepatites Virais [Internet]. Brasília: Ministério da Saúde; 2019 [citado 4 mar 2021]. Disponível em: http://www.aids.gov.br/pt-br/pub/2015/protocolo-clinicoe-diretrizes-terapeuticas-para-prevencao-da-transmissao-vertical-de-hiv 
4. Rocha KB, Santos RRG, Conz J, Silveira ACT. Transversalizando a rede: o matriciamento na descentralização do aconselhamento e teste rápido para HIV, sífilis e hepatites. Saúde Debate. 2016 jun;40(109):22-33. http://dx.doi.org/10.1590/0103-1104201610902.

5. Elko SP, McCartney E. Human immunodeficiency virus and pregnancy. Physician Assistant Clinics. 2018;3(3):399-410. http://dx.doi.org/10.1016/j. cpha.2018.02.008.

6. Sena AA, Alves dos Santos Bezerra MG, Araújo Almeida L. Occurrence of HIV and syphilis in the prenatal care of primary health care. Rev Pre Infec e Saúde. 2019;5:83512019. http://dx.doi.org/10.26694/repis. v5i0.8351.

7. Portaria $n^{\circ} 4.279$, de 30 de dezembro de 2010 (BR). Estabelece diretrizes para a organização da Rede de Atenção à Saúde no âmbito do Sistema Único de Saúde (SUS). Diário Oficial da União, Brasília (DF), 31 dez 2010: Seção 1: 89. Disponível em: http://bvsms.saude. gov.br/bvs/saudelegis/gm/2010/anexos/anexos_prt4279_30_12_2010. pdf

8. Viana ALA, Bousquat A, Melo GA, Negri A Fo, Medina MG. Regionalization and health networks. Ciênc Saúde Coletiva. 2018;23(6):1791-8.

9. Starfield B. Atenção primária: equilíbrio entre necessidades de saúde, serviços e tecnologia [Internet]. Brasília: Organização das Nações Unidas para a Educação, a Ciência e a Cultura/Ministério da Saúde; 2002 [citado 4 mar 2021]. Disponível em: https://www.nescon.medicina. ufmg.br/biblioteca/imagem/0253.pdf

10. Melo EA, Maksud I, Agostini R. Cuidado, HIV/Aids e atenção primária no Brasil: desafio para a atenção no Sistema Único de Saúde? Rev Panam Salud Publica. 2018;(42):e151. PMid:31093179.

11. Kleinubing RE, Eslava DG, Padoin SMM, Paula CC. Evaluation of the health attention to pregnant women with HIV: comparison between primary and specialized service. Esc Anna Nery. 2019;23(2):e20180258. http://dx.doi.org/10.1590/2177-9465-ean-2018-0258.

12. Rio Grande do Sul. Governo do Estado. Secretaria do Estado da Saúde. Coordenação Estadual de IST/AIDS. Coordenação Estadual de Atenção Básica. Linha de cuidado para pessoas vivendo com HIV (PVHIV) e outras infecções sexualmente transmissíveis. $2^{\mathrm{a}}$ ed. Porto Alegre (RS): Escola de Saúde Pública; 2018 [citado 4 mar 2021]. Disponível em: http://observatorioaids.saude.rs.gov.br/wp-content/uploads/2019/06/ Linha-de-Cuidado-E-book.pdf

13. Graham ID, Straus SE, Tetroe J. Knowledge translation in health care: moving from evidence to practice. 2nd ed. Chichester: John Wiley \& Sons. Canadá: Wiley Blackwell/BMJI Books; 2013. 424 p.

14. Vieira ACG, Gastaldo DHD, Harrison D. How to translate scientific knowledge into practice? Concepts, models and application. Rev Bras Enferm. 2020;73(5):e20190179. http://dx.doi.org/10.1590/0034-71672019-0179. PMid:32609213.

15. Kleinubing RE. Acessibilidade e utilização das redes de atenção primária e especializada por mulheres com HIV na perspectiva de usuárias e profissionais [tese]. Santa Maria (RS): Programa de Pós-graduação em Enfermagem, Universidade Federal de Santa Maria; 2018.

16. Kleinubing RE, Paula CC, Padoin SMM, Ferreira T, Silva FS. Accessibility and use of health services by HIV infected pregnant women: integrative review. Rev Baiana Enferm. 2017;31(2):e17437. http://dx.doi.org/10.18471/ rbe.v31i2.17437.

17. Kinalski DDF, Paula CC, Padoin SMM, Neves ET, Kleinubing RE, Cortes LF. Grupo focal na pesquisa qualitativa: relato de experiência. Rev Bras Enferm. 2017 abr;70(2):424-9. http://dx.doi.org/10.1590/0034-71672016-0091. PMid:28403311.

18. Minayo MCS. O desafio do conhecimento: pesquisa qualitativa em saúde. 14를 ed. São Paulo: Hucitec/ABRASCO; 2014.

19. Zambenedeth $\mathrm{G}$, Silva RAN. O paradoxo do território e os processos de estigmatização no acesso ao diagnóstico de HIV na atenção básica em saúde. Estud Psicol. 2015;20(4):229-40. http://dx.doi.org/10.5935/16784669.20150024
20. Garbin CAS, Martins RJ, Belila NM, Garbin AJI. The stigma of HIV positive users of the public health system. DST - J bras. Doenças Sex Transm. 2017;29(1):12-6. http://dx.doi.org/10.5533/DST-2177-8264201729104.

21. Gomes HN, Macena RHM, Arruda GMMS, Gomes AKCBP. Análise do atendimento nos serviços de saúde entre pessoas vivendo com HIV/AIDS. J. Health BiolSci. 2019;7(4):387-94. http://dx.doi.org/10.12662/23173076jhbs.v7i4.2595.p387-394.2019.

22. Massignani LRM, Rabuske MM, Backes MS, Crepaldi MA. Comunicação de diagnóstico de soropositividade HIV e aids por profissionais de saúde. Psicol Argum. 2014;32(79, Supl 2):65-75. http://dx.doi.org/10.7213/ psicol..argum.32.s02.AO06.

23. Colaço AD, Meirelles BHS, Heidemann ITSB, Villarinho MV. Care for the person who lives with HIV/AIDS in primary health care. Texto Contexto Enferm. 2019;28:e20170339. http://dx.doi.org/10.1590/1980-265xtce-2017-0339.

24. Nunes BP, Thumé E, Tomasi E, Duro SMS, Facchini LA. Socioeconomic inequalities in the access to and quality of health care services. Rev Saude Publica. 2014;48(6):968-76. http://dx.doi.org/10.1590/S0034 8910.2014048005388. PMid:26039400.

25. Silva LA, Alves VH, Rodrigues DP, Padoin SMM, Branco MBLR, Souza RMP. A qualidade de uma rede integrada: acessibilidade e cobertura no pré-natal. J Res Fundam Care. Online (Bergh). 2015;7(2):2298-309. http://dx.doi.org/10.9789/2175-5361.2015.v7i2.2298-2309.

26. Coelho B, Meirelles BHS. Care sharing for people with HIV/AIDS: a look targeted at young adults. Rev Bras Enferm. 2019;72(5):1341-8. http://dx.doi.org/10.1590/0034-7167-2018-0248. PMid:31531660.

27. Nakata LC, Feltrin AFS, Chaves LDP, Ferreira JBB. Conceito de rede de atenção à saúde e suas características-chaves: uma revisão de escopo. Esc Anna Nery. 2020;24(2):e20190154. http://dx.doi.org/10.1590/21779465-ean-2019-0154.

28. Ministério da Saúde (BR). Secretaria de Atenção à Saúde. Cinco passos para implementação do manejo da infecção pelo HIV na Atenção Básica [Internet]. Brasília: Ministério da Saúde; 2014. [citado 4 mar 2021]. Disponível em: http://www.aids.gov.br/pt-br/pub/2014/5-passos-paraimplementacao-do-manejo-da-infeccao-pelo-hiv-na-atencao-basica

29. Medeiros LB, Trigueiro DRSG, Silva DM, Nascimento JA, Monroe AA, Nogueira JA et al. Integração entre serviços de saúde no cuidado às pessoas vivendo com aids: uma abordagem utilizando árvore de decisão. Cien Saude Colet. 2016 fev;21(2):543-52. http://dx.doi. org/10.1590/1413-81232015212.06102015. PMid:26910161.

30. Costa JJ, Pinto ML, Prospero ENS, Lima RGS, Verdi M. Questões éticas implicadas no cuidado a gestantes portadoras de HIV. RBTS. 2016;3(1):41-52. http://dx.doi.org/10.14210/rbts.v3n1.p41-52.

31. Esposti CDD, Oliveira AE, Santos Neto ET, Travassos C. Representações sociais sobre o acesso e o cuidado pré-natal no Sistema Único de Saúde da Região Metropolitana da Grande Vitória, Espírito Santo. Saude Soc. 2015;24(3):765-79. http://dx.doi.org/10.1590/S0104-12902015127606.

32. Araújo CLF, Aguiar PS, Santos GKA, Oliveira MGP, Câmara LS. Anti-HIV testing in gynecology services in the city of Rio de Janeiro. Esc Anna Nery. 2014;18(1):82-9. http://dx.doi.org/10.5935/1414-8145.20140012.

33. Silva KS, Santos AM, Carvalho JA, Kochergin CN, De Almeida PF. Percepção de gestores e enfermeiros sobre a organização do fluxo assistencial na rede de serviços de saúde. RECIIS Rev Eletron Comun Inform Inov Saúde. 2017;11(2). http://dx.doi.org/10.29397/reciis.v11i2.1226.

34. Souza MHN, Nóbrega VM, Collet N. Social network of children with cronic disease: knowledge and practice of nursing. Rev Bras Enferm. 2020;73(2):e20180371. http://dx.doi.org/10.1590/0034-7167-20180371. PMid:32074233.

35. Silva AC, Schimint MD, Gomes TF, Weiller TH, Alberti GF, Simon BS Redes de atenção à saúde: percepção de usuários de uma unidade de saúde da família. Rev Enferm UFSM. 2017;7(1):100-10. http://dx.doi. org/10.5902/2179769225209.

\footnotetext{
a Artigo extraído da tese de doutorado de autoria de Raquel Einloft Kleinubing, defendida no ano de 2018, Programa de Pós-graduação em Enfermagem, Universidade Federal de Santa Maria, sob a orientação de Cristiane Cardoso de Paula. Texto completo disponível em: https://repositorio.ufsm.br/bitstream/ handle/1/18528/TES_PPGENFERMAGEM_2018_KLEINUBING_RAQUEL.pdf?sequence=1\&isAllowed=y
} 